\title{
The cost of operating room delays in an endourology center
}

Sara_Maskal, BSc ${ }^{1}$; Rajat Jain, $\mathrm{MD}^{2}$; Donald Fedrigon III, BSc ${ }^{1}$; Emily Rose, BSc ${ }^{3}$; Manoj Monga, $\mathrm{MD}^{2}$; Sri Sivalingam, $\mathrm{MD}^{2}$

${ }^{1}$ Case Western Reserve University School of Medicine, Cleveland, OH, United States; ${ }^{2}$ Cleveland Clinic Glickman Urological and Kidney Institute, Cleveland, $\mathrm{OH}$, United States; ${ }^{3}$ Cleveland Clinic Lerner College of Medicine of Case Western Reserve University, Cleveland, OH, United States

Cite as: Can Urol Assoc J 2020 February 4; Epub ahead of print. http://dx.doi.org/10.5489/cuaj.6099

Published online February 4, 2020

$* * *$

\section{Abstract}

Introduction: This study sought to characterize delays and estimate resulting costs during nephrolithiasis surgery.

Methods: Independent observers documented delays during ureteroscopy (URS) and percutaneous nephrolithotomy (PCNL) procedures. Fifty index cases over a period of three months was considered sufficient to observe the generalizable trends. Operating room staff, excluding the surgeons, were blinded. Time-related metrics and delays preventing case progression were recorded using a smartphone-accessible data collection instrument. Delays were categorized as: 1) missing equipment; 2) missing personnel; 3) equipment malfunction; or 4) delay due to case complexity. The first two categories were regarded as preventable and the latter two non-preventable.

Results: Forty URS and 18 PCNL cases were included. There was a total of 56 delays in 35 $(65 \%)$ cases. Twelve (67\%) PCNLs and 23 (58\%) URSs had delays $(\mathrm{p}=0.57)$. The mean cumulative delay per case was $3.5 \pm 3.2$ minutes. Pre-start delays $(n=17)$ were $4.5 \pm 3.5$ minutes on average while intraoperative delays $(n=39)$ were $3.1 \pm 2.9$ minutes $(\mathrm{p}=0.167)$. Delays were evenly spread among the four categories. Thirty-one (55\%) delays were preventable (mean $3.7 \pm 3.2$ minutes) while $25(45 \%)$ were non-preventable (mean $3.2 \pm 3.2$ minutes) $(\mathrm{p}=0.58)$. This translates to $\$ 137$ per case in preventable costs.

Conclusions: Preventable operative delays are encountered frequently in nephrolithiasis surgery, translating to significant additional charges and costs. We demonstrate a rationale for the development of improved communication and workflow protocols to increase efficiency in 
endourological surgeries. Key limitations are the observational nature of the study and sample size.

\section{Introduction}

Healthcare spending in the United States reached \$3.2 trillion in 2015. ${ }^{1}$ This has led to a heavy focus on improving the value of care and eliminating wastage. Operating room (OR) expenses can be a significant driver of medical costs. The CDC estimates that hospital discharges with a principal procedure in the operating room were responsible for $\$ 160$ billion worth of national costs in 2014 while Birkmeyer et al estimated that up to $40 \%$ of healthcare expenditure may revolve around surgical treatment. ${ }^{1,2}$ Baseline charges are $\$ 64-\$ 115$ per minute of OR time at our tertiary care center. This estimate does not include charges for anesthesia, drugs, supplies, physician services or anesthesia administration. Several studies have evaluated the cost of scopes, re-sterilization, disposable materials, but the direct impact of operating room costs has not been previously assessed in this specialty. ${ }^{3-8}$ Childers et al recently found the mean cost of operating time for acute care hospitals in California in 2014, defined as a combination of direct cost, wages and supplies, to be approximately $\$ 37 / \mathrm{min}$. ${ }^{9}$ We sought to characterize and quantify OR delays during procedures for treatment of nephrolithiasis at our high-volume stone center with the aim to guide future efforts to increase efficiency and minimize such delays.

\section{Methods}

All patients undergoing surgery for urolithiasis at a tertiary care stone referral center are prospectively enrolled into an institutional review board (IRB) approved stone registry. Specific review board approval for this study was not required as this data collection was classified as a quality assessment and improvement activity per institutional guidelines. Patients who underwent ureteroscopy (URS) and percutaneous nephrolithotomy (PCNL) procedures performed at two high volume, tertiary care sites at the Cleveland Clinic were identified. Patient demographics were recorded. An online smartphone-accessible data collection instrument was created through the REDCap (Research Electronic Data Capture) system which allowed for discrete time recording. ${ }^{10}$ Parameters were recorded in real time during the procedures. Two of the study investigators collected all of the data over a 3-month period. Given the nature of the study, it was determined that 50 index cases over a 3-month period was appropriate for showing generalizable trends in our surgeons' practices. All operating room staff were blinded to the study and purpose of observing personnel's presence excluding the two endourologists performing the procedures. Delays were defined as events that prevented case progression, specific potential scenarios and delay criteria were established prior to data collection. Delays were considered "pre-start" if they occurred after the patient entered the room and before scope insertion, which was considered to be the start of the procedure. "Intraoperative delays" occurred between the start and end of the procedure. 
There were no significant operational differences between the two endourologists. The equipment for the procedures was primarily stored in the operating room core area, and was brought into the room during case set up. Both surgeons provided the nursing team with lists of equipment to be opened prior to the case or available in the room and distance to stored equipment outside of the room was similar between the two sites. The OR team was comprised of a circulating genitourinary subspecialized nurse, one scrub nurse, a nurse anesthetist, anesthesiologist, the surgeon, and an endourology fellow. All patients were anesthetized using isoflurane and intubated with laryngeal mask airway or endotracheal tube at the anesthesiologist's discretion. No patients received spinal anesthesia.

During the data collection phase, delays were categorized as shown in Table 1, column 1. These categories were retrospectively refined into 4 groups: 1 - Missing or contaminated equipment, 2 - missing personnel, 3 - equipment malfunction, or 4 - interruptions due to case complexity or unexpected challenges (Table 1, column 2). The first two categories were regarded as preventable and the latter two were regarded as non-preventable. Our institution requires the attending anesthesiologist to be present for pre-procedure time-out and induction. Thus, delays due to anesthesiologist not being present were counted in category 2.

Estimates of charges were collected for CPT (current procedural terminology) codes for ureteroscopy and PCNL: 52352, 52353, 52356, 50080 and 50081 and based on level II-IV billing across both sites. At our institution, operating room charges varied based on case complexity and site. Ureteroscopy was charged at $\$ 64$ at the tertiary-level satellite hospital and $\$ 77 /$ minute at the main campus. Similarly, PCNL was charged at $\$ 92$ and $\$ 115 /$ minute, at each site, respectively. Given that charges can be variable between institutions, we utilized the OR cost estimate of $\$ 37 / \mathrm{min}$ as published by Childers et al in an effort to make our findings more generalizable. ${ }^{9}$

Statistical analysis was performed with two-tailed independent t-tests for continuous means and chi-square for categorical data. For all tests p-values $<0.05$ was considered statistically significant. Unless otherwise stated results are reported as mean \pm standard deviation. Statistical analysis was performed using R Statistical Software (R Foundation for Statistical Computing, Vienna, Austria).

\section{Results}

Recording was started for 44 URS and 18 PCNL cases. Three URS cases were excluded due to the data collector not being present for a significant portion of the case. Recording for one URS was aborted due to complication after 10 minutes while another was excluded because URS was attempted unsuccessfully and thus only a stent placement was performed. Forty URS and 18 PCNL were included in the analysis. The mean age of included patients was $60 \pm 15$ years. Mean operating time was $42.5 \pm 23.9$ minutes for URS and 63.7 \pm 31.2 minutes for PCNL. There were 56 delays in 35 (65\%) cases. Twelve (67\%) PCNLs and $23(58 \%)$ URSs had delays ( $\mathrm{p}=0.57)$. 
The overall mean length of delay was $3.5 \pm 3.2$ minutes. Mean length of delay for URS was 3.4 \pm 3.1 minutes or $8 \%$ of operating time. Mean length of delay for PCNL was $3.7 \pm 2.2$ minutes or $5.7 \%$ of operating time. Cumulative delay times per case ranged from 1:01-16:02 for URS and 00:47-7:43 for PCNL.

Thirty-one (55\%) delays were preventable (mean $3.7 \pm 3.2$ minutes) while $25(45 \%)$ were non-preventable (mean $3.2 \pm 3.2$ minutes) $(\mathrm{p}=0.55)$. The number of delays were evenly spread between the four categories: 16 (29\%), 15 (27\%), 12 (21\%), and 13 (23\%) delays, respectively.

Seventeen delays (30\%) were pre-start while 39 (70\%) were intraoperative. Pre-start delays were $4.5 \pm 3.5$ minutes on average while intraoperative delays were $3.1 \pm 2.9$ minutes $(\mathrm{p}=0.167)$. Of preventable delays, $14(45 \%)$ occurred pre-start and $17(55 \%)$ occurred intraoperatively. Of non-preventable delays, 3 (12\%) occurred pre-start and 22 (88\%) occurred intraoperatively. Preventable delays were more likely to occur pre-start than intraoperatively $(\mathrm{p}=0.009$, Table 2$)$.

Based on publicly reported charges for our institution and previously published OR costs, we estimated the additional charges and costs per case that were attributable to delays (Table 3). Patients undergoing URS incurred an additional mean charge of \$219-264 per case and patients undergoing PCNL incurred an additional mean charge of \$339-422 per case at our institution. In a more generalizable context, this translates to an added cost of $\$ 125$ and $\$ 135$ respectively. For URS patients, preventable delays constituted an additional mean charge of \$271while nonpreventable delays constituted an additional $\$ 191$. Similarly, this translates to an additional cost of \$141 and \$133 respectively. For PCNL patients, preventable delays constituted an additional mean charge of $\$ 375$ while non-preventable delays constituted an additional mean charge of $\$ 386$. This translates to an additional cost of $\$ 99$ and $\$ 137$ respectively. (Table 3)

\section{Discussion}

Previous reports such as that by Zygourakis et al suggest that awareness of OR equipment costs led to a $6.5 \%$ decrease in direct surgical supply costs. ${ }^{11}$ Surgical treatment of stone disease requires many pieces of reusable equipment as well as a large variety of disposable items such as wires, sheaths, and laser fibers. Cost and efficiency analyses of the surgical equipment, reprocessing and disposables has been reported previously. ${ }^{3-8}$ For example, one orthopedic study found that a dropped surgical instrument leads to a delay of 7.6 minutes. ${ }^{12}$ However, the direct charges associated with operating room delays have not been previously investigated in stone disease. A 2005 study of hospitals across the US found that operating room time averaged $\$ 62 /$ minute (range: $\$ 22-133 /$ minute) for all levels of case complexity. ${ }^{13}$ In this study, we sought to characterize and quantify delays and associated charges and costs arising in the OR during the surgical treatment of stone disease. By increasing awareness of this prevalent issue, we hope to provide a platform for improvements in efficiency and decreasing surgical costs. By 
characterizing these delays, we provide a platform to guide future interventions that could be implemented and studied to decrease OR delays and unnecessary costs.

We demonstrate that delays were encountered in the majority of routine endourological surgical cases. For ureteroscopy, the mean length of delay was 3.4 minutes, or almost $8 \%$ of the case length. This is a similar delay time compared to other studies. One observational study of delays in urological cases found a similar increase in operative time of 5.66 minutes in cases that lasted an average of 50 minutes. Another study of abdominal and orthopedic procedures found that surgical teams were distracted or interrupted 9.82 times per hour (standard deviation $=3.97$ ) in cases with a mean intraoperative duration was $1 \mathrm{~h}, 23 \mathrm{~min}$. Frequency of interrupting events, defined as events that halt a case for at least 10 seconds, was found to be higher in laparoscopic operations ( 0.38 per $\min )$ compared to open $(0.18$ per $\min )(p<0.001)$.

At our institution, an added 3.4 minutes translates to an average estimated unnecessary increase in charge of $\$ 242$ at our institution and average cost of $\$ 126$. Similarly, a mean length of delay of 3.7 minutes in PCNL translated to an average charge increase of \$381 and average cost of \$137. With an annual institutional caseload of over 400 URS and 400 PCNL the potential impact is $\$ 162,000$ in charges or $\$ 105,200$ in costs at our institution. There was no significant difference in the length of preventable and non-preventable delays. While the average delay may seem insignificant, they were significant in summation and contributed to turnover delays and significant subjective stress for the OR staff and surgeons.

The proportion of preventable delays was higher prior to the start of the procedures, which was similarly reported by Antoniadis et al. Additionally, intraoperative interruptions can lead to further miscommunications. These results suggest that clear protocols for communication between operating room personnel regarding equipment processing and set-up can potentially result in significant reduction in charges and improvement in efficiency, especially prior to the start of the procedure. As an example, one of our strategies has been the use of whiteboard magnets to delineate preoperatively what equipment is needed for each case (Figure 1).

Additionally, any intervention to prevent delays should address the issue of required personnel missing from the OR as this too, was a significant cause of delays. Surgical checklists have been used successfully as a tool to improve safety and reduce communication failures. ${ }^{14-16}$ We propose investigating the efficacy of a preoperative checklist to structure communication briefing regarding personnel and equipment needed in the OR. Use of such a tool prior to the patient entering the room would decrease delays as seen in our study population and increase overall OR efficiency. We also recognize that missing personnel is affected by adequacy of staffing and institutional culture. Addressing this issue will require interprofessional collaboration with nursing staff, anesthesia, and fluoroscopy technicians to identify underlying factors before we are able to implement quality improvement measures.

It is important to note that the majority of personnel working in these procedures were dedicated urology nurses and technologists, working in a high-volume tertiary referral center. As 
such, it is not unreasonable to expect these delays to be exaggerated for surgical centers where staff are not necessarily specialty-specific, or where staff experience with stone surgery is more limited than in our series.

This study was observational and only included two high volume endourologists. While turnover and effect on patient care are of interest, they could not be assessed with this study design. Turnover does not directly translate to an increased per-case charge and cost, but does lead to overall increased expenses for the surgical center due to resources required for a longer day, including more staff hours worked, overtime, etc. As the focus of this study was to evaluate surgical delays; estimates of charges excluded many important drivers of cost such as equipment, medication, personnel fees; these have been previously studied. ${ }^{3-6} \mathrm{We}$ did account for the variations in level of service charged, hence all the charges are given as a range, with the lower number representing a level II charge and the higher number representing a level IV charge (i.e. URS and PCNL, respectively), while cost was included as a more generalizable estimate.

\section{Conclusions}

In the surgical management of stone disease, delays are encountered in the majority of cases and appear to be preventable. This translated to a proportion of operative time leading to additional incurred charges and costs. We demonstrate a rationale and need for the development of improved communication and workflow protocols and dedicated operative team building to increase efficiency and prevention of delays in an endourological surgical setting. 


\section{References}

1. National Center for Health Statistics. Health, United States, 2016: With Chartbook on Long-Term Trends in Health. Hyattsville, MD; 2017. https://www.cdc.gov/nchs/data/hus/hus16.pdf\#056\%0A. Accessed May 31, 2019

2. Birkmeyer JD, Gust C, Baser O, Dimick JB, Sutherland JM, Skinner JS. Medicare payments for common inpatient procedures: implications for episode-based payment bundling. Health Serv Res 2010;45(6 Pt 1):1783-1795. doi:10.1111/j.14756773.2010.01150.x.

3. Del Santo K, Audouin M, Ouzaid I, Ravery V, Traxer O. Évaluation des résultats opératoires et des coûts liés à la mise en place d'une activité d'urétéroscopie souple au sein d'un centre hospitalo-universitaire. Progrès en Urol 2017;27(6):375-380. doi:10.1016/j.purol.2017.02.004.

4. Raheem OA, Khandwala YS, Sur RL, Ghani KR, Denstedt JD. Burden of Urolithiasis: Trends in Prevalence, Treatments, and Costs. Eur Urol Focus 2017;3(1):18-26. doi:10.1016/j.euf.2017.04.001.

5. San Juan J, Hou H, Ghani KR, Dupree JM, Hollingsworth JM. Variation in Spending around Surgical Episodes of Urinary Stone Disease-Findings from Michigan. J Urol November 2017. doi:10.1016/j.juro.2017.11.075.

6. Ziemba JB, Matlaga BR. Understanding the costs of flexible ureteroscopy. Minerva Urol Nefrol 2016;68(6):586-591. http://www.ncbi.nlm.nih.gov/pubmed/27364080. Accessed March 4, 2018.

7. Isaacson D, Ahmad T, Metzler I, et al. Defining the Costs of Reusable Flexible Ureteroscope Reprocessing Using Time-Driven Activity-Based Costing. J Endourol 2017;31(10):1026-1031. doi:10.1089/end.2017.0463.

8. Bagrodia A, Gupta A, Raman JD, Bensalah K, Pearle MS, Lotan Y. Predictors of Cost and Clinical Outcomes of Percutaneous Nephrostolithotomy. J Urol 2009;182(2):586590. doi:10.1016/j.juro.2009.04.014.

9. Childers CP, Maggard-Gibbons M. Understanding Costs of Care in the Operating Room. JAMA Surgery 2018; 153 (4). doi:10.1001/jamasurg.2017.6233

10. Harris PA, Taylor R, Thielke R, Payne J, Gonzalez N, Conde JG. Research electronic data capture (REDCap)--a metadata-driven methodology and workflow process for providing translational research informatics support. J Biomed Inform 2009;42(2):377381. doi:10.1016/j.jbi.2008.08.010.

11. Zygourakis CC, Valencia V, Moriates C, et al. Association Between Surgeon Scorecard Use and Operating Room Costs. JAMA Surg 2017;152(3):284. doi:10.1001/jamasurg.2016.4674.

12. Khan SA, Kumar A, Varshney MK, et al. Accidentally falling instruments during orthopaedic surgery: Time to wake up! ANZ J Surg 2008;78:794-5.

13. Macario A. What does one minute of operating room time cost? J Clin Anesth 2010;22(4):233-236. doi:10.1016/j.jclinane.2010.02.003.

14. Borchard A, Schwappach DLB, Barbir A, Bezzola P. A Systematic Review of the Effectiveness, Compliance, and Critical Factors for Implementation of Safety Checklists 
in Surgery. Ann Surg 2012;256(6):925-933. doi:10.1097/SLA.0b013e3182682f27.

15. Lingard L, Regehr G, Orser B, et al. Evaluation of a Preoperative Checklist and Team Briefing Among Surgeons, Nurses, and Anesthesiologists to Reduce Failures in Communication. Arch Surg 2008;143(1):12. doi:10.1001/archsurg.2007.21.

16. Treadwell JR, Lucas S, Tsou AY. Surgical checklists: a systematic review of impacts and implementation. BMJ Qual Saf 2014;23(4):299-318. doi:10.1136/bmjqs-2012-001797.

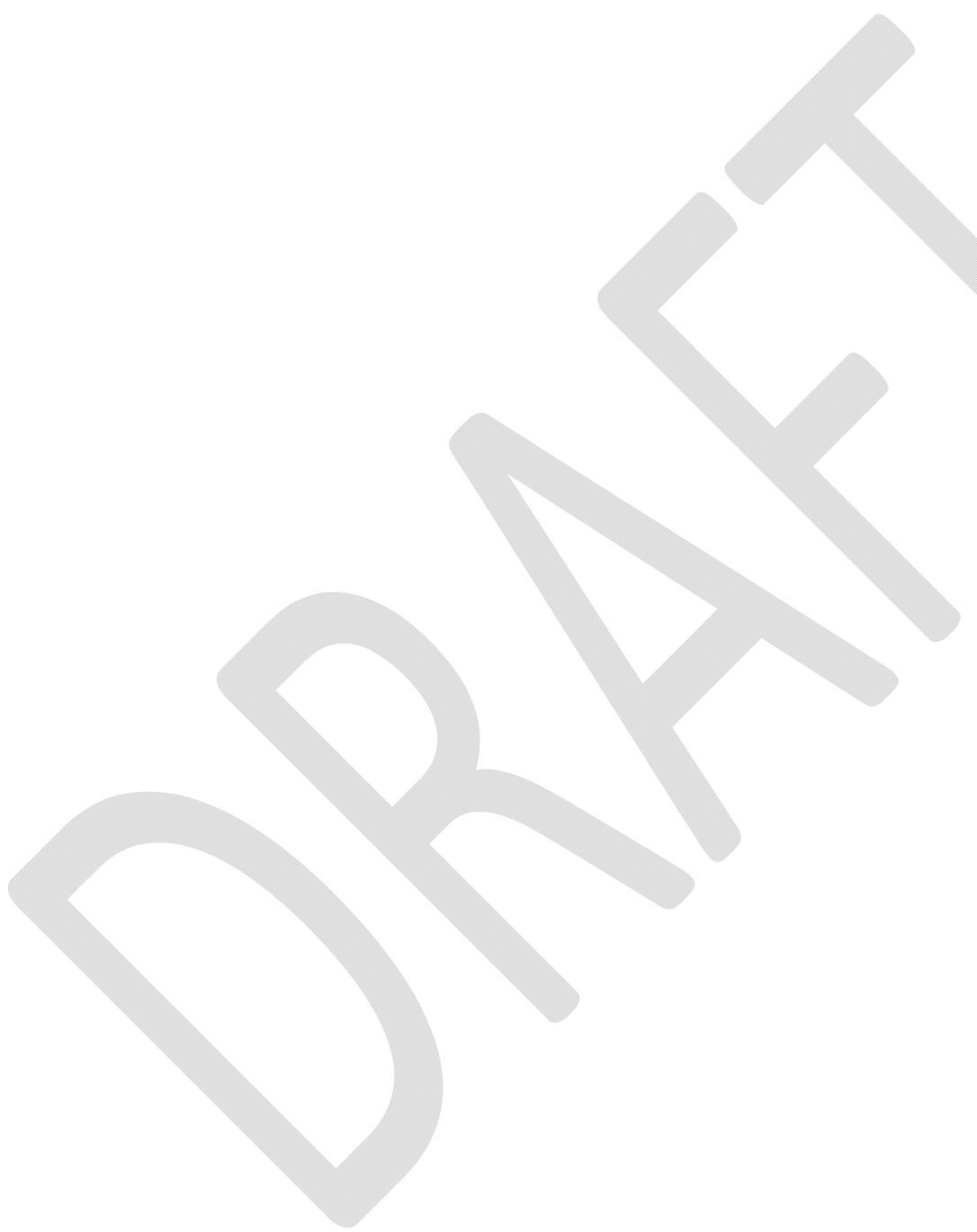


Figures and Tables

Fig. 1.

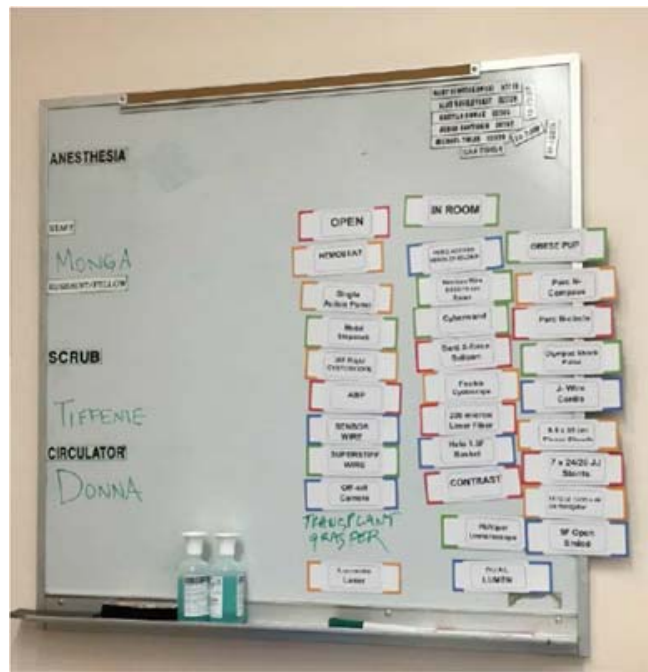

\section{Table 1. Delay categories}

\begin{tabular}{|c|c|c|c|c|}
\hline & $\begin{array}{l}\text { Data-collection } \\
\text { phase category }\end{array}$ & $\begin{array}{c}\text { Post data- } \\
\text { collection } \\
\text { phase } \\
\text { categories } \\
\end{array}$ & Preventable? & $\begin{array}{c}\text { Number of } \\
\text { delays per } \\
\text { category }\end{array}$ \\
\hline \multirow{3}{*}{ Pre-start delays } & $\begin{array}{c}\text { Anesthesia or surgeon } \\
\text { not available for } \\
\text { Huddle/Induction } \\
\end{array}$ & $\begin{array}{c}2-\text { missing } \\
\text { personnel }\end{array}$ & Yes & 4 \\
\hline & $\begin{array}{l}\text { Patient is positioned, } \\
\text { but fluoroscopy } \\
\text { equipment or } \\
\text { technician are not } \\
\text { present }\end{array}$ & $\begin{array}{c}1-\text { missing } \\
\text { equipment } \\
2-\text { missing } \\
\text { personnel }\end{array}$ & Yes & 5 \\
\hline & $\begin{array}{l}\text { Circulator required to } \\
\text { leave the room to } \\
\text { retrieve expected } \\
\text { equipment (video } \\
\text { tower, stirrups, etc.) }\end{array}$ & $\begin{array}{l}1-\text { missing } \\
\text { equipment }\end{array}$ & Yes & 2 \\
\hline
\end{tabular}




\begin{tabular}{|c|c|c|c|c|}
\hline & $\begin{array}{l}\text { Laser requested, but } \\
\text { personnel or } \\
\text { equipment is not in } \\
\text { room or personal } \\
\text { protective equipment } \\
\text { is missing }\end{array}$ & $\begin{array}{c}1-\text { missing } \\
\text { equipment } \\
2-\text { missing } \\
\text { personnel }\end{array}$ & Yes & 0 \\
\hline & $\begin{array}{l}\text { Event that causes a } \\
\text { delay but does not fit } \\
\text { into the other } \\
\text { categories }\end{array}$ & $1,2,3$, or 4 & $\begin{array}{c}\text { Attributed based } \\
\text { on debriefing } \\
\text { with surgeon }\end{array}$ & 6 \\
\hline \multirow{4}{*}{$\begin{array}{l}\text { Intraoperative } \\
\text { delay }\end{array}$} & $\begin{array}{l}\text { Expected equipment } \\
\text { is not in room (i.e., } \\
\text { guidewires, stents, } \\
\text { etc.) }\end{array}$ & $\begin{array}{l}1-\text { missing } \\
\text { equipment }\end{array}$ & & 13 \\
\hline & $\begin{array}{l}\text { Personnel (anesthesia, } \\
\text { nursing, fluoroscopy, } \\
\text { laser) are not in room }\end{array}$ & $\begin{array}{c}2-\text { missing } \\
\text { personnel }\end{array}$ & Yes & 6 \\
\hline & $\begin{array}{l}\text { Equipment that is not } \\
\text { in the room is } \\
\text { required due to } \\
\text { change in case plan or } \\
\text { equipment } \\
\text { malfunction }\end{array}$ & $\begin{array}{l}3 \text { - equipment } \\
\text { malfunction } \\
4 \text { - change in } \\
\text { case plan }\end{array}$ & No & 14 \\
\hline & $\begin{array}{l}\text { Event that causes a } \\
\text { delay but does not fit } \\
\text { into the other } \\
\text { categories }\end{array}$ & $1,2,3$, or 4 & $\begin{array}{c}\text { Attributed based } \\
\text { on debriefing } \\
\text { with surgeon }\end{array}$ & 6 \\
\hline
\end{tabular}

Table 2. Timing of preventable delays

\begin{tabular}{|l|c|c|c|}
\hline & Pre-start & Intraoperative & Total \\
\hline Preventable & $14(45 \%)$ & $17(55 \%)$ & 31 \\
\hline Non-preventable & $3(12 \%)$ & $22(88 \%)$ & 25 \\
\hline Total & $17(30 \%)$ & $39(70 \%)$ & 56 \\
\hline
\end{tabular}




\section{Table 3. Estimated additional charges to patients}

\begin{tabular}{|c|c|c|c|}
\hline & $\begin{array}{c}\text { Mean } \\
\text { length of } \\
\text { delay } \\
\text { (min:sec) }\end{array}$ & $\begin{array}{c}\text { Additional } \\
\text { charge in USD } \\
\text { (mean) }\end{array}$ & $\begin{array}{c}\text { Additional } \\
\text { mean cost in } \\
\text { USD }\end{array}$ \\
\hline URS & $3: 22$ & $\$ 219-264(242)$ & $\$ 125$ \\
\hline Preventable & $3: 49$ & $\$ 246-296(271)$ & $\$ 141$ \\
\hline Non-preventable & $2: 41$ & $\$ 173-208(191)$ & $\$ 99$ \\
\hline PCNL & $3: 39$ & $\$ 339-422(381)$ & $\$ 135$ \\
\hline Preventable & $3: 36$ & $\$ 334-416(375)$ & $\$ 133$ \\
\hline Non-preventable & $3: 42$ & $\$ 343-428(386)$ & $\$ 137$ \\
\hline
\end{tabular}

PCNL: percutaneous nephrolithotomy; URS: ureteroscopy. 